\title{
Generalised Stream X-Machines with Output Delimited Type
}

\author{
Tudor Bălănescu, \\ Faculty of Sciences, Piteşti University, Romania
}

\begin{abstract}
Some conditions relating to the automata involved in the W-testing method are discussed. It is also shown how to use the method for reduced automata instead of minimal automata. New design test conditions (weak output distinguishable, strong test-complete and output delimited type) are considered for the generalised stream $X$-machines (stream X-machines with basic functions replaced by relations and having as output strings of symbols rather than single symbols). It is proved that testing methods similar to those already developed for ordinary deterministic stream X-machines may be applied for generalised stream X-machines with output delimited types. A particular case of generalised stream X-machine with output delimited type is the X-machine with output delimiter, which produces outputs having a distinct right end character.
\end{abstract}

Keywords: Design for test conditions; Generalised stream X-machines; Output delimited type; Test completeness; Weak output distinguishable type; W-method

\section{Introduction}

Testing comes in several main forms, like structural or white box testing, functional or black box testing and statistical/random testing [HoI98] etc. Within these classes there are a number of specific methods and in some cases combinations of methods from more than one class. For any test method to be successful it has to be automated and in particular this involves the use of test generation tools, test application tools and test evaluation tools. Testing is a lengthy and expensive process, usually consuming more time and resources than the rest of the development process and in safety critical cases testing can amount to as much as $90 \%$ of the project. One of the reasons why structural testing, which is based on the structure of the implementation/code, is so widely practised is that often this is the only formal description available for feeding into a test generation tool. Statistical testing is based on an analysis of the potential usage and the likely input behaviour; this again can be used in the absence of formal specification. Functional testing does not rely on some formal specification. Some attempts are based on the use of a Z or VDM model; the technique usually used is the category partition method whereby the input domain is divided into natural classes and test values chosen randomly from these. There are systems, both hardware and software [Cho78], 
that can be expressed as a machine involving a finite number of internal states, a mechanism for the state to change when the system interacts with its environment and some mechanism for observing the results of these interactions. The concepts of input to and output from the system are important in developing a testing method. If we consider the final implementation of the desired system as being represented as a (usually unknown), machine of some sort, then the testing problem becomes one of carrying out experiments to establish whether the implementation machine behaves in the same way as the machine representing the specification of the desired system. These experiments involve the application of various sequences of system inputs into the implementation and the observation of the resultant outputs. The results from each experiment are then compared with the expected observations; if they are the same for all the experiments that are carried out then this may be used as evidence that the implementation may well satisfy its specification. Therefore, a testing method looking at the control structure of the software system and using a generalised finite state machine as the specification language is devised [Cho78]. The characteristics of this method are: only the control structure of the design is checked; it does not require an executable specification; and test sequences are guaranteed to reveal any errors in the control structure, provided that some reasonable assumptions are satisfied [Cho78]. In some applications we might find it convenient to specify the control structure such that the operation and the next state depend not only on the current state and a given input, but also on the status of certain global variables. In [Hol88, HoI98, IpH96] a new model is described which extends the finite state machine-based approach by considering the X-machine concept [Eil74]. An X-machine is like a finite state machine having in addition a basic data set, $X$ and a set of processing functions, $\Phi$ which operate on $X$. Each arrow in the finite state machine diagram is labelled by a function from $\Phi$. These functions are applied on an input data from $X$ in order to obtain an output, accordingly with the state transition given by the finite automaton. In the context of this model some data structures associated with certain operations described by the finite state machine structure may be introduced by using the concept of global memory [HoI98]. This new model, called stream X-machine [HoI98], passes over the limits issued with the finite state machine-based model. It contains a finite set of states $Q$, a finite input set $\Sigma$, and a finite output set $\Gamma$, just like a finite state machine. There is also an internal memory set $M$ (possibly infinite), which can take any convenient form. Given a current state and a current value for the memory, the machine processes as follows: reads an input symbol; moves to a new state; updates the memory; outputs some suitable value. The machine has an initial state and an initial memory value. It works on an input stream until this one is entirely scanned.

In [HoI98, IpH97] Chow's method for testing finite state automata is extended in order to test stream $\mathrm{X}$-machines. The clear advantage of this approach is that it guarantees that the system is fault free provided that the components are fault free.

In [BGG99] a special type of stream X-machines has been considered having their arcs labelled by processing relations instead of processing functions. This mechanism is obtained by coupling a stream Xmachine with a set of formal grammars describing processing relations. The power of these mechanisms and their connections with the cooperating distributed grammar systems controlled by graphs have been studied in [BGG99].

These kinds of stream X-machine with underlying distributed grammars leads to the concept of generalised stream $X$-machine [IpH99]. They are different from ordinary stream $\mathrm{X}$-machines in the following two aspects:

- the arcs are labeled by relations instead of functions;

- at each step it produces an output string instead of a single output symbol.

In [IpH99] the requirements have been studied to apply the testing methodology developed for stream $\mathrm{X}$-machines for generalised stream X-machines too.

In this paper we first show how Chow's approach may be used when some assumptions behind the automata theoretic method are relaxed and secondly we define new testing conditions for application to generalised stream X-machines with reduced associated automata and with output delimited types. These new testing conditions (weak output distinguishable and strong test complete type) lead to some more general methodologies for testing stream X-machines compared with those given in [HoI98, HBG00].

\section{Reduced and Minimal Automata}

Finite state machines have been a popular and effective way of specifying and designing a number of different types of systems. There are some advantages in using finite state machines as a specification language: they are intuitive, being based on a simple, dynamic model of computation; they can be represented in convenient 
ways such as diagrams or tables; and the theory of these machines is well known [HoI98]. The testing method dealing with a finite state machine model for the specification and implementation of software systems assumes that the machine is completely specified, minimal, with a fixed initial state and with every state reachable from the initial state [Cho78]. Sometimes the automata used in various examples or case studies [FBK90, FuB91] or the automata associated with some stream X-machines [HoI98] are not completely specified. In the sequel it is shown how Chow's method may be used for some relaxed conditions.

A (non-deterministic) finite automaton $\mathscr{A}$ over an alphabet $\Phi$ is a system $\left(Q, \Phi, F, q_{0}, T\right)$, where $Q$ is a finite, non-empty set of states, $\Phi$ is a finite input alphabet, and $F: Q \times \Phi \longrightarrow 2^{Q}$ is the next state function. Note that $F$ is a total function. For $q, q^{\prime} \in Q, \varphi \in \Phi$ we say that $q \stackrel{\varphi}{\rightarrow} q^{\prime}$ is an $\operatorname{arc}$ of $\mathscr{A}$ if $q^{\prime} \in F(q, \varphi)$. The function $F$ is usually described as a transition diagram, where the arcs corresponding to $F(q, \varphi)=\emptyset$ are not figured.

The mapping $F$ can be extended to the domain $Q \times \Phi^{*}$ by defining $F(q, \epsilon)=\{q\}$ ( $\epsilon$ is the empty sequence) and $F(q, x \varphi)=\bigcup_{p \in F(q, x)} F(p, \varphi), x \in \Phi^{*}, \varphi \in \Phi$. For $q, q^{\prime} \in Q, x \in \Phi^{*}$ we say that $q \stackrel{x}{\rightarrow} q^{\prime}$ is a path of $\mathscr{A}$ if $q^{\prime} \in F(q, x)$. The state $q_{0}$ is the initial state and $T \subseteq Q$ is the set of final states. By $\mathscr{L}(\mathscr{A})$ we denote the language accepted by $\mathscr{A}$. Two automata $\mathscr{A}$ and $\mathscr{A}^{\prime}$ are equivalent if $\mathscr{L}(\mathscr{A})=\mathscr{L}\left(\mathscr{A}^{\prime}\right)$. For an arbitrary set $K \subseteq \Phi^{*}, \mathscr{A}$ and $\mathscr{A}^{\prime}$ are called $K$ equivalent if $\mathscr{L}(\mathscr{A}) \cap K=\mathscr{L}\left(\mathscr{A}^{\prime}\right) \cap K$.

A deterministic automaton has a next state function $F$ such that $F(q, \varphi)$ contains at most one state for all $q \in Q, \varphi \in \Phi$. Considering that $F$ is undefined when $F(q, \varphi)=\emptyset$, we may view the next state function of a deterministic automaton as being a partial function $F: Q \times \Phi \longrightarrow Q$. A state $q$ is called accessible if there exists a path $q_{0} \stackrel{x}{\rightarrow} q$ from the initial state $q_{0}$ to $q$ (Definition 7.1.3, [HoI98]). An automaton is called accessible if all its states are accessible. Two states $q, p$ are called equivalent if for every path $q \stackrel{x}{\rightarrow} q^{\prime}$ there exists a path $p \stackrel{x}{\rightarrow} p^{\prime}$ and conversely (Definition 7.1.4, [HoI98]). A reduced automaton is a deterministic one having no equivalent distinct states (Definition 7.1.5, [HoI98]). For a given alphabet $\Phi$ and a language $L \subseteq \Phi^{*}$, the minimal automaton $M$ accepting $L$ is 'minimal' with respect to all deterministic automata accepting $L$ and having total next state functions of the form $F: Q \times \Phi \longrightarrow Q$, see [HoU69]. For a given language $L$, deterministic automata might exist with partial, next state function and having fewer states that the minimal automaton. These automata are reduced and accessible.

Example 1. For instance, if $\Phi=\{a, b\}$ and $L=\{\epsilon, a\}$ then the two-state automaton

$$
R=\left(\{0,1\},\{a, b\}, F_{1}, 0,\{0,1\}\right)
$$

where $F_{1}(0, a)=1$ and $F_{1}(q, \phi)$ is undefined otherwise, is not minimal.

Example 2. The three-state automaton

$$
M=\left(\{0,1, \text { trap }\},\{a, b\}, F_{2}, 0,\{0,1\}\right)
$$

where $F_{2}(0, a)=1, F_{2}(0, b)=F_{2}(1, a)=F_{2}(1, b)=F_{2}($ trap,$a)=F_{2}($ trap,$b)=$ trap is minimal.

A minimal automaton is reduced and accessible too. The automaton presented in Example 1 is reduced and accessible but it is not minimal.

Remark 1. If a minimal automaton accepting $L$ has $n$ states, then a reduced and accessible automaton $\mathscr{A}=\left(\Phi, Q, F, q_{0}, T\right)$ accepting $L$ has $n$ or $n-1$ states. Indeed, let us suppose that $\operatorname{card}(Q)=k$ and $k<n$. We consider the equivalent automaton $\mathscr{A}^{\prime}=\left(\Phi, Q \cup\{\operatorname{trap}\}, F^{\prime}, q_{0}, T\right)$ where trap $\notin Q$ is a new state and for all $q \in Q \cup\{\operatorname{trap}\}, F^{\prime}(q, \varphi)=\operatorname{trap}$ if and only if $F(q, \varphi)$ is undefined (note that $F^{\prime}(\operatorname{trap}, \varphi)=\operatorname{trap}$ ). Since $F^{\prime}$ is a total function, then $k+1 \geqslant n$, i.e. $k=n-1$.

In the sequel only deterministic automata will be considered and we want to decide the equivalence of two such automata using the concepts of test set and $k$-test set.

Definition 1. Test set for two automata. Let $\mathscr{A}=\left(\Phi, Q, F, q_{0}, T\right), \mathscr{A}^{\prime}=\left(\Phi, Q^{\prime}, F^{\prime}, q_{0}^{\prime}, T^{\prime}\right)$ be two finite automata over the same input alphabet $\Phi$. A test set of $\mathscr{A}$ and $\mathscr{A}^{\prime}$ is a finite set $K \subseteq \Phi^{*}$ such that $\mathscr{A}$ and $\mathscr{A}^{\prime}$ are equivalent if they are $K$-equivalent.

Definition 2. [HoI98] $k$-test set for an automaton. Let $\mathscr{A}=\left(\Phi, Q, F, q_{0}, T\right)$ be a finite automaton and $k \geqslant 0$ a natural number. A finite set $K \subseteq \Phi^{*}$ is a $k$-test set of $\mathscr{A}$ if it is a test set for $\mathscr{A}$ and any other finite state automaton $\mathscr{A}^{\prime}=\left(\Phi, Q^{\prime}, F^{\prime}, q_{0}^{\prime}, T^{\prime}\right)$ with $\operatorname{card}(Q) \leqslant \operatorname{card}\left(Q^{\prime}\right) \leqslant \operatorname{card}(Q)+k$. 
Remark 2. Based on these concepts, Chow [Cho78] introduces by the so-called $W$-method a way of building the above-mentioned finite sets. The two automata involved in testing their equivalence represent the description of the specification and the assumed implementation. The essential ideas are based around deriving sequences of inputs that can detect whether there are any extra states or missing states, and whether there are faulty or missing transitions in the implementation [HoI98]. Chow's W-testing method [Cho78] has been defined for minimal automata and it does not work for reduced and accessible automata.

Example 3. Let us consider for the specification the automaton given in Example 1 and for 'implementation' the automaton

$$
I=\left(\{0,1\},\{a, b\}, F_{3}, 0,\{0,1\}\right)
$$

where $F_{3}(0, a)=1, F_{3}(0, b)=1$ and undefined otherwise. We have that $S=\{\epsilon, a\}$ is a state cover set because any state can be reached from the initial state by choosing an appropriate input string of $S$. We have also that $W=\{a\}$ is a characterisation set for it because for any $q, q^{\prime} \in Q$ there exists an element of $W$ which is accepted when starting from $q$ and rejected when starting from $q^{\prime}$. Since the automaton and its implementation have the same number of states, the $\mathrm{W}$-method gives the 0-test set $K=S \Phi W \cup S W=\{a, a a, b a, a a a, a b a\}$. However, the two automata are $K$-equivalent but they are not equivalent. Indeed, the single word which distinguishes them is $b$ but it is not a member of $K$.

Note that for the real minimal automaton from Example 2 we must have $\epsilon \in S$ (it is the only way to attend the initial state 0 ) and $\epsilon \in W$ (it is the only word which distinguishes the state 1 from the state trap.) It follows that $b \in K$ and the two automata are not K-equivalent.

In practice, the reduced automata are very often used instead of minimal ones, due to the fact that the number of transition arrows is significantly lower. The W-method may be used for reduced and accessible automata too, keeping in mind that there is an 'invisible' trap state in the diagram of the automaton.

Proposition 1. If $S$ is a state cover set and $W$ is a characterisation set of a reduced and accessible automaton, then a $k$-test set $K^{\prime}$ is constructed using the same formula as in the $\mathrm{W}$-method [Cho78], i.e. $K^{\prime}=S^{\prime}\left(\Phi^{k+1} \cup \ldots\{\epsilon\}\right) W^{\prime}$ where $S^{\prime}=S \Phi \cup S$ and $W^{\prime}=W \cup\{\epsilon\}$.

Proof. If the reduced and accessible automaton is not minimal, then the minimal one is obtained by adding the new state trap (see Remark 1.) We use $S^{\prime}$ instead of $S$ in order to simulate that the trap state is attended ( $S^{\prime}$ is in fact a transition cover for the reduced and accessible automaton). We use also $\epsilon \in W^{\prime}$ in order to distinguish between the trap state and any other final state with no arrow emerging from it.

\section{Design for Test Conditions for Stream X-Machines}

In order to get the most from a testing strategy certain constraints must be imposed. In hardware design the idea of design for test is well understood. If the designer ignores the fact that the system will eventually need testing, thorough testing is very hard, if not impossible. The same is true for software; the conditions imposed by the stream X-machines and presented in the sequel represent a formalisation of the idea of design for test.

\subsection{Basic Definitions}

Definition 3. [HoI98] A stream X-machine (SX-machine, for short) $\mathscr{M}$ is a tuple

$$
\left(\Sigma, \Gamma, Q, M, \Phi, F, q_{0}, T, m_{0}\right)
$$

where:

- $\Sigma$ and $\Gamma$ are finite sets called the input and output alphabet respectively;

- $Q$ is the finite set of states;

- $M$ is a (possibly) infinite set called memory;

- $\Phi$ is the type of $\mathscr{M}$, a finite set of basic functions of the form

$$
\varphi: M \times \Sigma \longrightarrow \Gamma \times M
$$

- $F: Q \times \Phi \longrightarrow 2^{Q}$ is the next state (partial) function; 
- $q_{0} \in Q$ is the initial state and $T \subseteq Q$ is the set of terminal states;

- $m_{0} \in M$ is the initial memory value.

Note 1. If $\mathscr{M}=\left(\Sigma, \Gamma, Q, M, \Phi, F, q_{0}, T, m_{0}\right)$ is an SX-machine, then $\mathscr{A}=\left(\Phi, Q, F, q_{0}, T\right)$ is called the associated automaton of $\mathscr{M}$. The arcs and the paths of $\mathscr{A}$ are the arcs and the paths of $\mathscr{M}$ too.

When a given basic function operating in a given state with a memory value and an input element produces an output symbol then there is no other basic function producing the same output symbol under identical conditions. These conditions are expressed by the output distinguishability property.

Definition 4. [HoI98] Output distinguishable type. A type $\Phi$ is called output distinguishable if for all $\varphi, \varphi^{\prime} \in \Phi$ we have that $\varphi=\varphi^{\prime}$ if there exist $m \in M, \sigma \in \Sigma$ and $g \in \Gamma$ such that $\varphi(m, \sigma)=\left(g, m_{1}\right), \varphi^{\prime}(m, \sigma)=\left(g, m_{1}^{\prime}\right)$.

Note 2. For a (partial) function $\varphi: A \longrightarrow B$, we denote by $\operatorname{domain}(\varphi)$ the set of all elements $a \in A$ such that $\varphi(a)$ is defined.

Definition 5. [HoI98] Test-complete type. A processing function $\varphi \in \Phi$ is called test complete with respect to the memory $M$ (t-complete) if for all $m \in M$ there exists $\sigma_{\varphi, m} \in \Sigma$ such that $\left(m, \sigma_{\varphi, m}\right) \in \operatorname{domain}(\varphi)$.

A type $\Phi$ is called test complete with respect to the memory $M$ if all the functions of $\Phi$ are test complete with respect to $M$.

This condition prevents the 'dead-ends' in the machine. It may be observed that any stream X-machine may be turned into a test-complete one by introducing a new input symbol and by extending the functions that are not test complete by defining them for these new input symbols and for any memory value.

In order to introduce the relation computed by an SX-machine, it may be observed that the machine in the initial state and processing an input sequence of elements starting from the initial memory value might produce an output sequence of symbols. The functions involved in each such processing lead to a so-called path function.

Definition 6. Path function. Each path $p=\varphi_{1} \ldots \varphi_{n}, n \geqslant 1, \varphi_{i} \in \Phi$ gives rise to a function (the path function)

$$
|p|: M \times \Sigma^{*} \rightarrow \Gamma^{*} \times M
$$

where $|p|(m, s)=\left(g, m^{\prime}\right)$ if and only if there exist $m_{2}, \ldots, m_{n+1} \in M$ and $\forall i, 1 \leqslant i \leqslant n, \varphi_{i}\left(m_{i}, \sigma_{i}\right)=\left(g_{i}, m_{i+1}\right)$, where $m_{1}=m, m_{n+1}=m^{\prime}, s=\sigma_{1} \ldots \sigma_{n}, g=g_{1} \ldots g_{n}$. The function corresponding to the empty path $\epsilon$ is $|\epsilon|(m, \epsilon)=(\epsilon, m)$, for any $m \in M$.

Definition 7. Computed relation. [HoI98] Given an SX-machine $\mathscr{M}$, we define the relation

$$
f: \Sigma^{*} \leftrightarrow \Gamma^{*}
$$

by

$$
s f g(\text { or } g \in f(s))
$$

if and only if

$$
\exists q \in T, m^{\prime} \in M
$$

and a path $q_{0} \stackrel{p}{\longrightarrow} q$ with $|p|\left(m_{0}, s\right)=\left(g, m^{\prime}\right)$.

We say that $f$ is the relation computed by $\mathscr{M}$. For a subset $Y \subseteq \Sigma^{*}$, we denote by $f_{\mid Y}$ the restriction of $f$ to $Y$, i.e. $g \in f_{\mid Y}(s)$ if and only if $g \in f(s)$ and $s \in Y$.

The basis for testing an SX-machine is to check the internal structure of the associated automaton by means of some strings which are able to exercise every function in any given reachable state. The next definition deals with this concept.

Definition 8. [IpH99] Test function. Let $\mathscr{M}=\left(\Sigma, \Gamma, Q, M, \Phi, F, q_{0}, T, m_{0}\right)$ be an SX-machine. A function $t: \Phi^{+} \longrightarrow \Sigma^{+}$is called a test function of $\mathscr{M}$ if for any $p \in \Phi^{+}$the value $t(p)$ satisfies the following properties:

- if $p$ is a path in $\mathscr{M}$ starting in $q_{0}$ then $\left(m_{0}, t(p)\right) \in \operatorname{domain}(|p|)$

- if $p=\varphi_{1} \ldots \varphi_{n}$ is not a path of $\mathscr{M}$, then $\left(m_{0}, t(p)\right) \in \operatorname{domain}\left(\left|\varphi_{1} \ldots \varphi_{k} \varphi_{k+1}\right|\right)$, where $\varphi_{1} \ldots \varphi_{k}, 0 \leqslant k<n$ is the longest prefix of $p$ which is a path in $\mathscr{M}$ starting in $q_{0}$. 
In order to derive a method for testing that two SX-machines compute the same relation, a test set of the two SX-machines is defined as being a set of input sequences of which successful application to the two SX-machines will lead to the conclusion of the equivalence of these mechanisms. This is the way the W-testing method developed by [Cho78] was extended to SX-machines in [HoI98].

Definition 9. [HoI98] Test set for two stream $X$-machines. Let $\mathscr{M}=\left(\Sigma, \Gamma, Q, M, \Phi, F, q_{0}, T, m_{0}\right), \mathscr{M}^{\prime}=$ $\left(\Sigma, \Gamma, Q^{\prime}, M, \Phi, F^{\prime}, q_{0}^{\prime}, T^{\prime}, m_{0}\right)$ be two stream X-machines having the same type and the same sets of input symbols, output symbols and memory. Let $f: \Sigma^{*} \leftrightarrow \Gamma^{*}, f^{\prime}: \Sigma^{*} \leftrightarrow \Gamma^{*}$ be the relations computed by $\mathscr{M}, \mathscr{M}^{\prime}$ respectively. A set $Y \subseteq \Sigma^{*}$ is a test set for $\mathscr{M}, \mathscr{M}^{\prime}$ if from $f_{\mid Y}=f_{\mid Y}^{\prime}$ it follows that $f=f^{\prime}$.

Definition 10. [HoI98] $k$-test set for a stream $X$-machine. Let $\mathscr{M}=\left(\Sigma, \Gamma, Q, M, \Phi, F, q_{0}, T, m_{0}\right)$ be a stream $\mathrm{X}$-machine and $k \geqslant 0$ a natural number. A set $Y \subseteq \Sigma^{*}$ is a $k$-test set for $\mathscr{M}$ if it is a test set for $\mathscr{M}$ and any other stream X-machine $\mathscr{M}^{\prime}=\left(\Sigma, \Gamma, Q^{\prime}, M, \Phi, F^{\prime}, q_{0}^{\prime}, T^{\prime}, m_{0}\right)$ with $\operatorname{card}(Q) \leqslant \operatorname{card}\left(Q^{\prime}\right) \leqslant \operatorname{card}(Q)+k$.

If the associated automaton of a stream X-machine is minimal, then according to Chow's result [Cho78] the automaton has a k-test set $K$. In addition, it is known from [HoI98] that if the type of the stream $\mathrm{X}$-machine is test complete with respect to memory, then it has a test function $t$. Moreover, if the type is output distinguishable then $t(K)$ is a $\mathrm{k}$-test set for the given $\mathrm{X}$-machine.

\subsection{New Design for Test Conditions for Generalised Stream X-Machines}

The generalised stream X-machines are introduced in [IpH99]. They are stream X-machines which have the arcs representing relations instead of functions. Moreover, the relations have as output strings of symbols instead of single symbols. We introduce here more general design for test conditions and prove that the test methodology developed in [HoI98] may be applied in this case too. In the sequel are defined the concepts of generalised stream $X$-machine as well as those corresponding to path relation and relation computed by such a mechanism.

Definition 11. [IpH99] A generalised stream $X$-machine (GSX-machine, for short) $\mathscr{M}$ is a tuple

$$
\left(\Sigma, \Gamma, Q, M, \Phi, F, q_{0}, T, m_{0}\right)
$$

where all the elements are as in Definition 3 except the type $\Phi$, which is defined as follows:

- $\Phi$, the type of $\mathscr{M}$, is a set of basic non-empty relations that the machine can use, of the form:

$$
\varphi: M \times \Sigma \longleftrightarrow \Gamma^{*} \times M
$$

Note 3. GSX-machines are stream X-machines for which the computational process is based on relations rather than functions. This kind of mechanism is used in [BGG99] where the relations associated with the derivation process are defined by some production rules. In order to change its configuration, the GSXmachine will read an input symbol, discard it and produce a sequence of output symbols while (possibly) changing the value of the memory. For a given input symbol and a memory value the machine may produce zero or many output sequences. If there is at most one output sequence and its length is one, i.e. the processing relations are in fact functions of the form

$$
\varphi: M \times \Sigma \longrightarrow \Gamma \times M
$$

then the GSX-machine is a stream $X$-machine (SX-machine).

It may be seen that for a GSX-machine processing an input sequence may lead to more possible output sequences. hence, the next definition deals with path relations instead of path functions.

Definition 12. Path relation. Let $\mathscr{M}$ be a GSX-machine. Each path $p=\varphi_{1} \ldots \varphi_{n}, n \geqslant 1, \varphi_{i} \in \Phi$ gives rise to a relation (the path relation)

$$
|p|: M \times \Sigma^{*} \longleftrightarrow \Gamma^{*} \times M
$$

where $(m, s)|p|\left(g, m^{\prime}\right)$ if and only if there exist $m_{2}, \ldots, m_{n} \in M$ and $\forall i, 1 \leqslant i \leqslant n,\left(m_{i}, \sigma_{i}\right) \varphi_{i}\left(g_{i}, m_{i+1}\right)$, where $m_{1}=m$ and $m_{n+1}=m^{\prime}$ and $s=\sigma_{1} \ldots \sigma_{n}, g=g_{1} \ldots g_{n}$. The relation corresponding to the empty path $\epsilon$ is $(m, \epsilon)|\epsilon|(\epsilon, m)$, for any $m \in M$. 
Definition 13. Computed relation. Given a GSX-machine $\mathscr{M}$, we define the relation

$$
f: \Sigma^{*} \longleftrightarrow \Gamma^{*}
$$

by

$s f g$

if and only if

$$
\exists q_{0} \in I, q \in T, m^{\prime} \in M
$$

and a path $q_{0} \stackrel{p}{\longrightarrow} q$ with $\left(m_{0}, s\right)|p|\left(g, m^{\prime}\right)$.

We say that $f$ is the relation computed by $\mathscr{M}$. For a subset $Y \subseteq \Sigma^{*}$, we denote by $f_{\mid Y}$ the restriction of $f$ to $Y$, i.e. $s f_{\mid Y} g$ if and only if $s f g$ and $s \in Y$.

A machine computation takes the form of a path traversal in the state space and the application, in turn, of the path labels (which represent basic processing relations) to the initial memory value. The correspondence between the input sequence applied to the machine and the output produced gives rise to the relation computed by the machine. In general, a GSX-machine is non-deterministic, in the sense that the application of an input sequence can produce more than one single output sequence.

\subsection{Weak Output Distinguishable and Strong Test Complete Types}

In this section we introduce variants of the design for test conditions presented in [HoI98]. More precisely, the concepts of output distingushability for relations, weak output distinguishability and strong test completeness also for relations are defined.

Definition 14. [IpH99] A type $\Phi$ containing relations $\varphi: M \times \Sigma \longleftrightarrow \Gamma^{*} \times M$ is output distinguishable if for all $\varphi, \varphi^{\prime} \in \Phi$ we have

$$
\varphi=\varphi^{\prime} \text { and } m_{1}=m_{1}^{\prime}
$$

if there exists $\sigma \in \Phi, m \in M$ and $g \in \Gamma^{*}$ such that

$$
(m, \sigma) \varphi\left(g, m_{1}\right),(m, \sigma) \varphi^{\prime}\left(g, m_{1}^{\prime}\right)
$$

Looking at Definition 4, it may be observed that in this case not only the simple replacing of functions by relations is performed but additionally it is imposed that the resulting computed value of the memory is also the same.

Note 4. If $\Phi$ contains only functions, then Definitions 14 and 4 are equivalent. Indeed, $m_{1}=m_{1}^{\prime}$ follows from $\varphi=\varphi^{\prime}$. This is not true if $\Phi$ contains relations. For instance, let us consider $\Sigma=\{0\}, \Gamma=\{a\}, M=\{\alpha, \beta$, and $\Phi=\{\omega\}$, where

$$
\omega(\alpha, 0)=\{(a, \alpha),(a, \beta)\}
$$

The type $\Phi$ is not output distinguishable. Indeed, for $\varphi=\varphi^{\prime}=\omega$ we have $(\alpha, 0) \varphi(a, \alpha)$ and $(\alpha, 0) \varphi^{\prime}(a, \beta)$ but $\alpha \neq \beta$.

In the next definition, the output distinguishability property imposed in Definition 4 is relaxed by considering that each relation $\varphi \in \Phi$ can be distinguished in the set $\Phi$ by a specific input symbol $\sigma_{\varphi}$.

Definition 15. A type $\Phi$ containing relations $\varphi: M \times \Sigma \longleftrightarrow \Gamma^{*} \times M$ is weak output distinguishable if for all $\varphi \in \Phi$ there exists an input symbol $\sigma_{\varphi} \in \Sigma$ such that for all $\varphi^{\prime} \in \Phi$, we have

$$
\varphi=\varphi^{\prime} \text { and } m_{1}=m_{1}^{\prime}
$$

if there exist $m \in M$ and $g \in \Gamma^{*}$ such that

$$
\left(m, \sigma_{\varphi}\right) \varphi\left(g, m_{1}\right),\left(m, \sigma_{\varphi}\right) \varphi^{\prime}\left(g, m_{1}^{\prime}\right)
$$


Example 4. Let us consider a GSX-machine with $\Sigma=\{0,1\}, \Gamma=\{a, b\}, M=\{\alpha, \beta, \gamma\}$ and $\Phi=\left\{\varphi_{1}, \varphi_{2}\right\}$, where

$$
\begin{array}{ll}
\varphi_{1}(\alpha, 0)=(a, \alpha) & \varphi_{2}(\alpha, 0)=(a, \alpha) \\
\varphi_{1}(\beta, 0)=(b, \alpha) & \varphi_{2}(\beta, 0)=(a, \alpha) \\
\varphi_{1}(\gamma, 0)=(b, \alpha) & \\
\varphi_{1}(\alpha, 1)=(b, \alpha) & \varphi_{2}(\alpha, 1)=(a, \alpha) \\
\varphi_{1}(\beta, 1)=(a, \alpha) & \varphi_{2}(\beta, 1)=(b, \alpha) \\
& \varphi_{2}(\gamma, 1)=(b, \alpha)
\end{array}
$$

The type $\Phi$ is weak output distinguishable. We have $\sigma_{\varphi_{1}}=\sigma_{\varphi_{2}}=1$.

Lemma 2. If the type $\Phi$ is output distinguishable then it is weak output distinguishable.

Proof. Indeed, let us consider an arbitrary $\varphi \in \Phi$. It is supposed that $\operatorname{domain}(\varphi) \neq \emptyset$, otherwise $\varphi$ is a useless function of $\Phi$ and will be removed. We may take as $\sigma_{\varphi}$ any input symbol such that there exists an $m \in M$ and $\left(m, \sigma_{\varphi}\right) \in \operatorname{domain}(\varphi)$.

Lemma 3. There are weak output distinguishable types which are not output distinguishable.

Proof. Let us consider the type from Example 4. Since $\varphi_{1}(\alpha, 0)=\varphi_{2}(\alpha, 0)$, but $\varphi_{1} \neq \varphi_{2}$, the type is not output distinguishable.

These two results show that the class of GSX-machines having output distinguishable types is strictly included in the set of GSX-machines with weak output distinguishable types. The same observation may be mentioned for SX-machines too.

Now, for weak output distinguishable types a new test completeness condition, stronger than that given by Definition 5, is given.

Definition 16. Let $\Phi$ be a weak output distinguishable type. It is called strong test complete with respect to the memory $M$ if

$$
\forall \varphi \in \Phi, \forall m \in M\left(\left(m, \sigma_{\varphi}\right) \in \operatorname{domain}(\varphi)\right)
$$

where $\sigma_{\varphi}$ is as in Definition 15 .

Lemma 4. A strong test-complete type is test complete and there are test-complete types which are not strong test complete.

Proof. The first fact follows from Definitions 5 and 16. The type from Example 4 is not strong type complete because $\varphi_{1}(\gamma, 1)$ is undefined.

In [HoI98] it is shown how a test function can be constructed for a test-complete SX-machine. Similarly it may be considered a test function for a strong test complete GSX-machine.

Lemma 5. Let $\mathscr{M}=\left(\Sigma, \Gamma, Q, M, \Phi, F, q_{0}, m_{0}\right)$ be a GSX-machine. If $\Phi$ is strong test complete with respect to $M$, then there exists a test function for $\mathscr{M}$.

Proof. We define st : $\Phi^{+} \longrightarrow \Sigma^{+}$as follows:

$\operatorname{st}(\varphi)=\sigma_{\varphi}$ for all $\varphi \in \Phi$, and

$$
s t(p \varphi)= \begin{cases}s t(p) \sigma_{\varphi} & \text { if } p \text { is a path } \\ s t(p), & \text { otherwise }\end{cases}
$$

for $p \in \Phi^{+}$and $\varphi \in \Phi$. Using the fact that $\Phi$ is strong test complete, it may be proved by induction on the length of the path $p$ that $s t(p)$ has the properties from Definition 8.

Note 5. We say that st is the strong test function of the machine $\mathscr{M}$. If $\varphi_{1} \ldots \varphi_{k}$ is a path of $\mathscr{M}$ and $\varphi_{1} \ldots \varphi_{k} \varphi$, $\varphi \in \Phi$ is not a path of $\mathscr{M}$ then $s t\left(\varphi_{1} \ldots \varphi_{k} \varphi \alpha\right)=\sigma_{\varphi_{1}} \ldots \sigma_{\varphi_{k}} \sigma_{\varphi}$ for any $\alpha \in \Phi^{*}$.

The notions of test set for two GSX-machines and k-test set for a GSX-machine are similar to the notions from Definitions 9 and 10 considering the relations $f: \Sigma^{*} \longleftrightarrow \Gamma^{*}, f^{\prime}: \Sigma^{*} \longleftrightarrow \Gamma^{*}$ instead of the functions $f: \Sigma^{*} \longrightarrow \Gamma^{*}, f^{\prime}: \Sigma^{*} \longrightarrow \Gamma^{*}$. 


\subsection{Output Delimited Type}

A testing strategy is introduced for those GSX-machines satisfying the condition that the strings of output symbols produced by a relation may be identified in any arbitrary identical sequences of strings of output symbols.

Definition 17. A weak output distinguishable type $\Phi$ is called an output delimited type if for any two strings of relations $\varphi_{1} \ldots \varphi_{j}$ and $\varphi_{1}^{\prime} \ldots \varphi_{j}^{\prime} \in \Phi^{*}$, any two output strings $x_{1} \ldots x_{j}$ and $y_{1} \ldots y_{j}$ with each $x_{i}, y_{i} \in \Gamma^{*}$, from

- $x_{1} \ldots x_{j}=y_{1} \ldots y_{j}$

- $\left(x_{1}, m_{1}\right) \in \varphi_{1}\left(m_{0}, \sigma_{\varphi_{1}}\right),\left(y_{1}, m_{1}^{\prime}\right) \in \varphi_{1}^{\prime}\left(m_{0}, \sigma_{\varphi_{1}}\right)$,

- $\left(x_{i}, m_{i},\right) \in \varphi_{i}\left(m_{i-1}, \sigma_{\varphi_{i}}\right),\left(y_{i}, m_{i}^{\prime},\right) \in \varphi_{i}^{\prime}\left(m_{i-1}^{\prime}, \sigma_{\varphi_{i}}\right)$ for all $2 \leqslant i \leqslant j$

we have that $x_{i}=y_{i}$, for all $1 \leqslant i \leqslant j$.

Example 5. The type $\Phi=\left\{\varphi_{1}, \varphi_{2}\right\}$ with $\varphi_{1}\left(m_{0}, 0\right)=\left(a, m_{0}\right), \varphi_{2}\left(m_{0}, 0\right)=\left(a a, m_{0}\right)$ is a weak output distinguishable type, where $\sigma_{\varphi_{1}}=\sigma_{\varphi_{2}}=0$. It is not an output delimited type; indeed, $\varphi_{1} \varphi_{2}$ and $\varphi_{2} \varphi_{1}$ produce the same output $a a a$ when processing the input $\sigma_{\varphi_{1}} \sigma_{\varphi_{2}}=00$ but we have $x_{1}=a, y_{1}=a a$.

It follows that for an output delimited type an arbitrary path produces the same output as $\varphi_{1} \ldots \varphi_{j}$ processing the same input $\sigma_{\varphi_{1}} \ldots \sigma_{\varphi_{j}}$ only if it produces the same output as $\varphi_{i}$ at each stage $i, 1 \leqslant i \leqslant j$. Any weak output distinguishable type $\Phi=\{\varphi: M \times \Sigma \longleftrightarrow \Gamma \times M\}$ containing only relations producing single symbols is an output delimited type.

Therefore the ordinary stream X-machines having an weak output distinguishable type are stream Xmachines with output delimited types. More generally, any weak output distinguishable type of the form $\Phi=\left\{\varphi \mid \varphi: M \times \Sigma \longleftrightarrow \Gamma^{m} \times M\right\}, m \geqslant 1$ is an output delimited type.

In [HBG00] a particular case of output delimited type is described, namely the type with output delimiter. A GSX-machine with output delimiter works like a GSX-machine, but changing the configuration it writes a string of symbols which ends always with a special output symbol, named output delimiter.

Definition 18. [HBG00] A GSX-machine with output delimiter is a GSX-machine $\mathscr{M}=\left(\Sigma, \Gamma, Q, M, \Phi, F, q_{0}, T, m_{0}\right)$, where all the relations of the type $\Phi$ have the form $\varphi: M \times \Sigma \longrightarrow\left(\Gamma^{*}\{\tau\}\right) \times M$, and $\tau \notin \Gamma$. The element $\tau$ is called the output delimiter of the machine.

The testing strategy for a GSX-machine with a reduced and accessible associated automaton and having output delimited and strong test complete type is based on the next result.

Lemma 6. Let $\mathscr{M}=\left(\Sigma, \Gamma, Q, M, \Phi, F, q_{0}, T, m_{0}\right)$, and $\mathscr{M}^{\prime}=\left(\Sigma, \Gamma, Q^{\prime}, M, \Phi, F^{\prime}, q_{0}^{\prime}, T^{\prime}, m_{0}\right)$ be two GSX-machines, having the same elements $\Sigma, \Gamma, M, \Phi, m_{0}$. Let $f, f^{\prime}$ be the two relations they compute and $\mathscr{A}, \mathscr{A}^{\prime}$ their associated automata.

If $\Phi$ is an output delimited and strong test-complete type with respect to memory then for any set $K \subseteq \Phi^{*}$ we have

$$
\left(f_{\mid s t(K)}=f_{\mid s t(K)}^{\prime}\right) \Longrightarrow \mathscr{L}(\mathscr{A}) \cap K=\mathscr{L}\left(\mathscr{A}^{\prime}\right) \cap K
$$

where $s t: \Phi^{*} \longrightarrow \Sigma^{*}$ is the strong test function of $\mathscr{M}$.

Proof. Note that since the type is strong test complete with respect to memory it follows from Lemma 5 that there exists the strong test function st for $\mathscr{M}$.

We prove the lemma by contradiction, that is, we assume that $f_{\mid s t(K)}=f_{\mid s t(K)}^{\prime}$ and $\mathscr{L}(\mathscr{A}) \cap K \neq \mathscr{L}\left(\mathscr{A}^{\prime}\right) \cap K$. Now two cases are possible.

Case 1. There exists a path $\varphi_{1} \ldots \varphi_{n} \in K \cap\left(\mathscr{L}(\mathscr{A}) \backslash \mathscr{L}\left(\mathscr{A}^{\prime}\right)\right), n \geqslant 1$. We have that $\operatorname{st}\left(\varphi_{1} \ldots \varphi_{n}\right)=\sigma_{\varphi_{1}} \ldots \sigma_{\varphi_{n}} \in$ $s t(K)$. Since the type is strong test complete with respect to memory, then there exists some outputs $g_{1}, \ldots g_{n} \in \Gamma^{*}$ and memory values $m_{1}, \ldots m_{n} \in M$ such that $\left(g_{i}, m_{i}\right) \in \varphi_{i}\left(m_{i-1}, \sigma_{\varphi_{i}}\right)$ for all $1 \leqslant i \leqslant n$. Hence $g_{1} \ldots g_{n} \in f\left(\sigma_{\varphi_{1}} \ldots \sigma_{\varphi_{n}}\right)$. It follows from $f_{\mid s t(K)}=f_{\mid s t(K)}^{\prime}$ that $g_{1} \ldots g_{n} \in f^{\prime}\left(\sigma_{\varphi_{1}} \ldots \sigma_{\varphi_{n}}\right)$. Then we have a path $\varphi_{1}^{\prime} \ldots \varphi_{n}^{\prime} \in \mathscr{L}\left(\mathscr{A}^{\prime}\right)$, the memory values $m_{1}^{\prime} \ldots m_{n}^{\prime}$ and the outputs $g_{1}^{\prime}, \ldots g_{n}^{\prime} \in \Gamma^{*}$ such that $\left(g_{1}^{\prime}, m_{1}^{\prime}\right) \in \varphi_{i}^{\prime}\left(m_{0}, \sigma_{\varphi_{1}}\right)$ $\left(g_{i}^{\prime}, m_{i}^{\prime}\right) \in \varphi_{i}^{\prime}\left(m_{i-1}^{\prime}, \sigma_{\varphi_{i}}\right)$ for all $2 \leqslant i \leqslant n$ and $g_{1} \ldots g_{n}=g_{1}^{\prime}, \ldots g_{n}^{\prime}$. Since the type $\Phi$ is output delimited, it follows that $g_{i}=g_{i}^{\prime}$ for all $1 \leqslant i \leqslant n$. Since the type is weak output distinguishable, it follows that $\varphi_{i}^{\prime}=\varphi_{i}$ for all $1 \leqslant i \leqslant n$; note that the condition $m_{1}=m_{1}^{\prime}$ from Definition 15 is used in proving that. Hence $\varphi_{1} \ldots \varphi_{n} \in \mathscr{L}\left(\mathscr{A}^{\prime}\right)$, a contradiction. 
Case 2. There exists a path $\varphi_{1}^{\prime} \ldots \varphi_{n}^{\prime} \in K \cap\left(\mathscr{L}\left(\mathscr{A}^{\prime}\right) \backslash \mathscr{L}(\mathscr{A})\right), n \geqslant 1$. We have that $\operatorname{st}\left(\varphi_{1}^{\prime} \ldots \varphi_{n}^{\prime}\right)=\sigma_{\varphi_{1}^{\prime}} \ldots \sigma_{\varphi_{j}^{\prime}} \sigma_{\varphi_{j+1}^{\prime}} \in$ $s t(K)$ where $0 \leqslant j \leqslant n-1, \varphi_{1}^{\prime} \ldots \varphi_{j}^{\prime} \in \mathscr{L}(\mathscr{A})$ and $\varphi_{1}^{\prime} \ldots \varphi_{j}^{\prime} \varphi_{j+1}^{\prime} \notin \mathscr{L}(\mathscr{A})$. Since $\varphi_{1}^{\prime} \ldots \varphi_{j}^{\prime} \varphi_{j+1}^{\prime}$ is a path in $\mathscr{A}^{\prime}$ and the type is strong test complete it follows that there exists $g_{1}^{\prime} \ldots g_{j}^{\prime} g_{j+1}^{\prime} \in \Gamma^{*}, m_{1}^{\prime}, \ldots, m_{j}^{\prime}, m_{j+1}^{\prime} \in M$ such that $\left(g_{1}^{\prime}, m_{1}^{\prime}\right) \in \varphi_{1}^{\prime}\left(m_{0}, \sigma_{\varphi_{1}^{\prime}}\right),\left(g_{i}^{\prime}, m_{i}^{\prime}\right) \in \varphi_{i}^{\prime}\left(m_{i-1}^{\prime}, \sigma_{\varphi_{i}^{\prime}}\right)$, for all $2 \leqslant i \leqslant j+1$. Since $f_{\mid s t(K)}=f_{\mid s t(K)}^{\prime}$ it follows that there exists a path $\varphi_{1} \ldots \varphi_{j} \varphi_{j+1}$ in $\mathscr{A}$, the outputs $g_{1} \ldots g_{j} g_{j+1} \in \Gamma^{*}$ and the memory values $m_{1}, \ldots m_{j}, m_{j+1} \in M$ such that $g_{1} \ldots g_{j} g_{j+1}=g_{1}^{\prime} \ldots g_{j}^{\prime} g_{j+1}^{\prime}$ and $\left(g_{i}, m_{i}\right) \in \varphi_{i}\left(m_{i-1}, \sigma_{\varphi_{i}^{\prime}}\right)$, for all $1 \leqslant i \leqslant j+1$. Since the type is output delimited, it follows that $g_{i}=g_{i}^{\prime}$ for all $1 \leqslant i \leqslant j+1$. Since the type is weak output distinguishable, we have that $\varphi_{i}=\varphi_{i}^{\prime}$ for all $1 \leqslant i \leqslant j+1$. Hence, $\varphi_{1}^{\prime} \ldots \varphi_{j}^{\prime} \varphi_{j+1}^{\prime}$ is a path in $\mathscr{A}$, a contradiction.

Theorem 7. If $\mathscr{M}=\left(\Sigma, \Gamma, Q, M, \Phi, F, q_{0}, m_{0}\right)$ is a GSX-machine such that:

1. its associated automaton $\mathscr{A}$ is reduced and accessible;

2. its type $\Phi$ is output delimited and strong test complete memory;

then for any $k \geqslant 0$ it has a $k$-test set.

Proof. According to Proposition 1 there exists a $k$-test set $K$ for the automaton $\mathscr{A}$. It follows from Lemma 6 that $\operatorname{st}(K)$ is a $k$-test set of $\mathscr{M}$.

Remark 3. The hypotheses of the Theorem 7 are slightly different from those stated by Theorem 7.3.1 in [HoI98]. Indeed, the associated automaton is reduced and accessible and the type is weak output distinguishable. These requirements are weaker than those stated in [HoI98], where the automaton is minimal and the type is output distinguishable. However, in Theorem 7 the type must be strong complete; even though this is a stronger requirement, it may be easily fulfilled by extending the input alphabet $\Sigma$ to $\Sigma_{\xi}=\Sigma \cup\{\xi\}$, the output alphabet to $\Gamma_{\xi}=\Gamma \cup\left\{o_{\varphi} \mid \varphi \in \Phi\right\}$ and every processing function $\varphi \in \Phi$ to $\varphi_{\xi}$, where $\xi \notin \Sigma$ is a new input symbol, $o_{\varphi} \neq o_{\varphi^{\prime}}$ if $\varphi \neq \varphi^{\prime}, \varphi_{\xi}(m, \sigma)=\varphi(m, \sigma)$ for $\sigma \in \Sigma$ and $\varphi_{\xi}(m, \xi)=\left(o_{\varphi}, m\right)$ for all $m \in M$. Using the symbol $\xi$ the type becomes both weak output distinguishable and strong test complete.

Remark 4. The type $\Phi$ of any GSX-machine with output delimiter is output delimited. Hence, Theorem 7 holds for any GSX-machines with output delimiter and strong test complete type.

Remark 5. Let us consider a GSX-machine as in Theorem 7. The generation of a k-test set consists of the following steps:

1. Construct a state cover $S$ and a characterisation set $W$ for the associated automaton.

2. If the associated automaton is reduced and accessible but not minimal, then compute $S^{\prime}=S \Phi \cup S$ and $W^{\prime}=W \cup\{\epsilon\}$; otherwise, $S^{\prime}=S, W^{\prime}=W$.

3. Generate a k-test set of the associated automaton $K^{\prime}=S^{\prime}\left(\Phi^{k+1} \cup \ldots\{\epsilon\}\right) W^{\prime}$ where $S^{\prime}=S \Phi \cup S$ and $W^{\prime}=W \cup\{\epsilon\}$.

4. Convert $K^{\prime}$ to $X=s t\left(K^{\prime}\right)$, the k-test set for the given GSX machine.

Example 6. Let us consider a GSX-machine having the input alphabet $\Sigma=\{0,1,2\}$, the memory $M=\left\{m_{0}, m_{1}\right\}$, the output alphabet $\Gamma=\{a, b\}$ and the type $\Phi=\left\{\varphi_{0}, \varphi_{1}, \varphi_{2}\right\}$, where the processing functions are given in the following tables:

\begin{tabular}{cccc}
\hline$\varphi_{0}$ & 0 & 1 & 2 \\
\hline$m_{0}$ & $\left(a b, m_{1}\right)$ & $\left(a a, m_{1}\right)$ & $\left(a b, m_{1}\right)$ \\
$m_{1}$ & $\left(b a, m_{1}\right)$ & $\left(a b, m_{1}\right)$ & $\left(a a, m_{1}\right)$ \\
\hline
\end{tabular}




\begin{tabular}{cccc}
\hline$\varphi_{1}$ & 0 & 1 & 2 \\
\hline$m_{0}$ & $\left(b a, m_{1}\right)$ & $\left(a b, m_{1}\right)$ & $\left(a b, m_{1}\right)$ \\
$m_{1}$ & $\left(a b, m_{1}\right)$ & $\left(a a, m_{1}\right)$ & $\left(a a, m_{1}\right)$ \\
\hline
\end{tabular}

\begin{tabular}{cccc}
\hline$\varphi_{2}$ & 0 & 1 & 2 \\
\hline$m_{0}$ & $\left(b a, m_{1}\right)$ & $\left(b a, m_{1}\right)$ & $\left(a a, m_{1}\right)$ \\
$m_{1}$ & $\left(a b, m_{1}\right)$ & $\left(a b, m_{1}\right)$ & $\left(a b, m_{1}\right)$ \\
\hline
\end{tabular}

The type is output delimited and strong test complete, where $\sigma_{\varphi_{0}}=0, \sigma_{\varphi_{1}}=1, \sigma_{\varphi_{2}}=2$. The associated automaton is

$$
A=\left(\{0,1\},\left\{\phi_{0}, \phi_{1}, \phi_{2}\right\}, F, 0,\{0,1\}\right)
$$

where $F\left(0, \phi_{0}\right)=F\left(0, \phi_{1}\right)=0, F\left(0, \phi_{2}\right)=F\left(1, \phi_{2}\right)=1$.

We use the methodology from Remark 5 in order to compute a 0 -test set for this GSX machine. We obtain $S=\left\{\epsilon, \varphi_{2}\right\}, W=\{\epsilon\}$. Since the automaton is not minimal, we compute $S^{\prime}=\left\{\epsilon, \varphi_{0}, \varphi_{1}, \varphi_{2}, \varphi_{2} \varphi_{0}, \varphi_{2} \varphi_{1}, \varphi_{2} \varphi_{2}\right\}$ and $W^{\prime}=\{\epsilon\}$.

Next, a 0 -test set of the associated automaton is obtained:

$$
\begin{aligned}
K^{\prime}= & \left\{\epsilon, \varphi_{0}, \varphi_{1}, \varphi_{2}, \varphi_{0} \varphi_{0}, \varphi_{0} \varphi_{1}, \varphi_{0} \varphi_{2}, \varphi_{1} \varphi_{0}, \varphi_{1} \varphi_{1}, \varphi_{1} \varphi_{2}, \varphi_{2} \varphi_{0}, \varphi_{2} \varphi_{1}, \varphi_{2} \varphi_{2},\right. \\
& \left.\varphi_{2} \varphi_{0} \varphi_{0}, \varphi_{2} \varphi_{0} \varphi_{1}, \varphi_{2} \varphi_{0} \varphi_{2}, \varphi_{2} \varphi_{1} \varphi_{0}, \varphi_{2} \varphi_{1} \varphi_{1}, \varphi_{2} \varphi_{1} \varphi_{2}, \varphi_{2} \varphi_{2} \varphi_{0}, \varphi_{2} \varphi_{2} \varphi_{1}, \varphi_{2} \varphi_{2} \varphi_{2}\right\}
\end{aligned}
$$

Finally, the 0 -test set of the given GSX-machine is:

$$
X=s t\left(K^{\prime}\right)=\{0,1,2,00,01,02,10,11,12,20,21,22,200,201,202,210,211,212,220,221,222\}
$$

\section{Conclusions}

In this paper a testing strategy is defined for (generalised) stream X-machines which is based on Chow's method for finite state machines [Cho78] and on a testing method developed by Holcombe and Ipate [HoI98] and further improved in [HBG00]. The method of generating test sequences is a generalisation of that presented in [HoI98] and requires output delimited and strong test complete as design for test conditions. The first condition is weaker than that imposed in [HoI98]. Even though the second one is stronger than test completeness, it may be easily fulfilled by extending the input alphabet and the type of generalised stream $\mathrm{X}$-machine. In a forthcoming paper we shall use similar extensions in order to generate test sequences for a larger class of generalised stream X-machines.

\section{References}

[BGG99] Bălănescu, T., Gheorghe, M. and Georgescu, H.: Stream X-Machines with underlying distributed grammars. Informatica, 1999 (submitted).

[Cho78] Chow, T. S.: Testing software design modeled by finite-state machines. IEEE Transactions on Software Engineering, 4(3): 178-187, 1978.

[Eil74] Eilenberg, S.: Automata, Languages and Machines. Academic Press, London, 1974.

[FBK90] Fujiwara, S., Bochmann, G. v., Khendek, F., Amalou, M. and Ghedamsi, A.: Test selection based on finite state models. Publication \#716, Departement d'informatique et de recherche operationnelle, University of Montreal, 1990.

[FuB91] Fujiwara, S. and Bochmann, G. v.: Testing non-deterministic finite state machines. Publication \#758, Departement d'informatique et de recherche operationnelle, University of Montreal, 1991.

[Hol88] Holcombe, M.: X-machines as a basis for dynamic system specification. Software Engineering Journal, 3: 69-76, 1988.

[HoI98] Holcombe, M. and Ipate F.: Correct Systems: Building a Business Process Solution. Springer, Berlin, 1998. 
[HBG00] Holcombe, M., Bălănescu, T., Gheorghe, M. and Radovici-Mărculescu, P.: On testing generalized stream X-machines. In G. Păun, editor, Recent Topics in Mathematical and Computational Linguistics. Editura Academiei Romane, 130-141, 2000. [HoU69] Hopcroft, J. E., and Ullman, J. D.: Formal Languages and their Relation to Automata. Addison-Wesley, Reading, MA, 1969. [IpH96] Ipate, F. and Holcombe, M.: Another look at computability. Informatica, 20: 359-372, 1996.

[IpH97] Ipate, F. and Holcombe, M.: An Integration Testing Method That is Proved to Find all Faults. International Journal of Computer Mathematics, 69: 159-178, 1997.

[IpH99] Ipate, F. and Holcombe, M.: Generating test sequences from non-deterministic X-machines. Unpublished manuscript, 1999.

Received October 2000

Accepted January 2001 